\title{
HNX/SuperTIGER Silicon Strip Detector Response to Nuclei in Lead Primary and Fragmented Test Beams
}

\author{
John F. Krizmanic ${ }^{* 1,2,3 \dagger}$, Yosui Akaike ${ }^{1,2,3}$, Dana Braun ${ }^{4}$, Richard Bose ${ }^{4}$, James \\ Buckley $^{4}$, Georgia De Nolfo ${ }^{2}$, Jeff Dumonthier ${ }^{2}$, Paul Dowkontt ${ }^{4}$, Zachary Hughes ${ }^{4}$, \\ Iker Liceaga Indart ${ }^{2,10}$, Jason Link ${ }^{1,2,3}$, John W. Mitchell ${ }^{2}$, J. Grant Mitchell ${ }^{2,11}$, \\ Martin A. Olevitch ${ }^{4}$, Scott Nutter ${ }^{5}$, Garry E. Simburger ${ }^{4}$, George Suarez ${ }^{2}$, Teresa \\ Tatoli $^{1,2,3}$, Mark E. Wiedenbeck ${ }^{6}$, W. Robert Binns ${ }^{4}$, Theresa .J. Brandt ${ }^{2}$, Martin H. \\ Israel $^{4}$, Allan W. Labrador ${ }^{7}$, Richard A. Mewaldt ${ }^{7}$, Brian F. Rauch ${ }^{4}$, Kenichi Sakai ${ }^{1,2,3}$, \\ Makoto Sasaki ${ }^{1,2,8}$, Edward C. Stone ${ }^{6}$, Nathan E. Walsh ${ }^{4}$, C. Jake Waddington ${ }^{9}$ \\ ${ }^{1}$ Center for Research and Exploration in Space Science \& Technology \\ ${ }^{2}$ NASA/Goddard Space Flight Center, Greenbelt, MD, USA \\ ${ }^{3}$ University of Maryland, Baltimore County, Baltimore, MD, USA \\ ${ }^{4}$ Dept. of Physics, Washington University in St. Louis, St. Louis, MO, USA \\ ${ }^{5}$ Dept. of Physics, Northern Kentucky University, Highland Heights, KY, USA \\ ${ }^{6}$ Jet Propulsion Laboratory, California Institute of Technology, Pasadena, CA, USA \\ ${ }^{7}$ Space Radiation Lab, California Institute of Technology, Pasadena, CA, USA \\ ${ }^{8}$ University of Maryland, College Park, College Park, MD USA \\ ${ }^{9}$ School of Physics \& Astronomy, University of Minnesota, Minneapolis, MN, USA \\ ${ }^{10}$ Catholic University of America, Washington, DC, USA \\ ${ }^{11}$ Dept. of Physics, George Washington University, Washington, DC, USA
}

\begin{abstract}
The response to $150 \mathrm{GeV} /$ nuc primary lead $\left({ }^{208} \mathrm{~Pb}\right)$ and fragmented $(\mathrm{A} / \mathrm{Z}=2.4,2.2,2.0)$ beams measured a silicon strip detector, designed for use in the Heavy Nuclei eXplorer (HNX) and an upgrade of the Super Trans-Iron Galactic Element Recorder (SuperTIGER) balloon experiment, was evaluated in a CERN test beam (H8A) during Nov - Dec 2018. The $500 \mu \mathrm{m}$ thick, singlesided silicon detectors have 32 DC-coupled strips with $3 \mathrm{~mm}$ pitch on the junction side with $9.6 \times 9.6 \mathrm{~cm}^{2}$ active area. Discrete charge-preamplifiers and shaping amplifiers were used to read out the ohmic and junction side signals simultaneously using the SuperTIGER DAQ system. We report on the response in a configuration where all 32 strips were joined and read out together. The strip detector-under-test was situated between planar silicon detectors, which provided the charge selection as well as a comparison of the measured response of each detector. The combined data set shows excellent charge resolution and finely resolved elemental peaks from helium $(Z=2)$ through lead $(\mathrm{Z}=82)$. In this paper, we provide a description of the test beam experiment and the results of the charge resolution analysis.
\end{abstract}

36th International Cosmic Ray Conference -ICRC2019-

July 24th - August 1st, 2019

Madison, WI, U.S.A.

\footnotetext{
* Speaker.

${ }^{\dagger}$ Corresponding Author: E-mail: john.f.krizmanic@ @nasa.gov
} 


\section{Introduction}

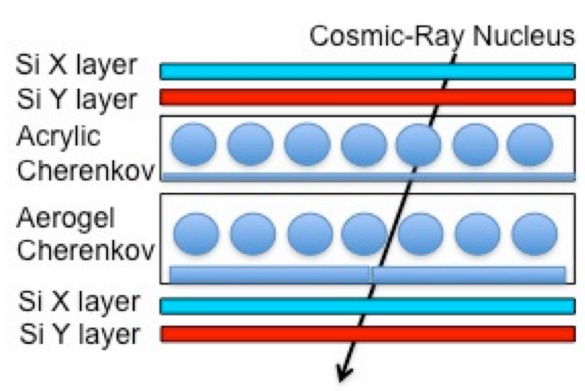

Figure 1: A schematic of the CosmicTIGER instrument illustrating the SSD and Cherenkov detector subsystems.
The Heavy Nuclei eXplorer (HNX) mission [1, $2,3]$ is comprised of two instruments to measure the individual cosmic ray elemental abundances from carbon through curium in a two year mission. The Extremely-heavy Cosmic-ray Composition Observer (ECCO) employs $\sim 21 \mathrm{~m}^{2}$ of barium phosphate (BP-1) glass tiles to record individual $\mathrm{CR}$ nuclei with $Z \geq 70$ with high sensitivity through the actinides. ECCO, based on the Trek experiment [4], requires the BP1 tiles to be returned to the Earth for processing. The Cosmic-ray Trans-Iron Galactic Element Recorder (CosmicTIGER) is an electronic instrument with sufficient dynamic range to measure cosmic-ray (CR) nuclei from carbon through curium, but with an area of $2 \mathrm{~m}^{2}$. Thus CosmicTIGER has a large measurement overlap with ECCO, especially in the $70 \leq Z \leq 82$ region where event statistics are sufficiently large. CosmicTIGER employs three detector subsystems: silicon strip detector (SSD) arrays, an acrylic $(n=1.5)$ Cherenkov detector, and an aerogel $(\mathrm{n}=1.04)$ Cherenkov detector. The Cherenkov detectors provide measurement of cosmic ray charge and velocity with two different energy thresholds. The SSD array provides the charge measurements with excellent charge resolution while also providing measurement of individual nuclei trajectories. These allow the identification of CR nuclei at the individual element level via the use of the $d E / d x$ versus Cherenkov and Cherenkov versus Cherenkov techniques.

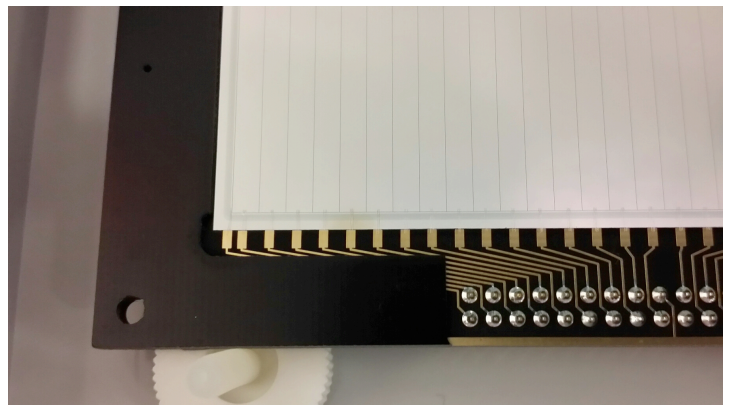

Figure 2: The strip side of a corner of an SSD mounted on its window-pane board (from Ref. [7]).

A schematic of CosmicTIGER is shown in Figure 1, showing the four planes of SSDs in a orthogonal $\mathrm{x}-\mathrm{y}$ arrangement above and below the Cherenkov detectors, Each SSD plane is a mosaic of $10 \times 20$ SSDs each with $\sim 10 \times 10 \mathrm{~cm}^{2}$ active area. Each SSD is a single-sided DC-coupled silicon strip detector with 32 channels with $3 \mathrm{~mm}$ pitch on the junction side and are $500 \mu \mathrm{m}$ thick, The current design has ten SSDs daisy-chained together to form ladders, with each ladder being readout by a two PHASIC ASICs [5] providing both tracking and charge measurement of the traversing CR. The ohmic side of each SSD will be read out by a discrete charge-preamplifier chain to provide precision charge measurement and event triggering signals. Based on the performance of the SSD and electronics, the anticipated charge resolution is $\sigma_{Z}<0.25$ up to $\mathrm{Z}=96$. The SDD detectors developed for HNX could be employed in an upgrade in the long duration balloon (LDB) SuperTIGER instrument [6] to provide both the tracking an charge measurements.

Five prototype single-sided, DC-coupled HNX/SuperTIGER SSD detectors, each with active area of $96 \times 96 \mathrm{~mm}^{2}$, were fabricated by Micron Semiconductors, LTD. Each detector was mounted 
to a window pane circuit board, with the SSD strip, guard ring, field plate, and ohmic side channels wire bonded to traces connected to a two row-pin connector on opposite ends of the board. Figure 2 shows the corner of a strip side of a mounted SSD. Each of the SSDs fully deplete $\lesssim 32 \mathrm{~V}$ with a total leakage current (32 strips + guard ring) of $\sim 100 \mathrm{nA}$ at $100 \mathrm{~V}$ and at room temperature. The individual strip leakage currents for each SSD was determined to be $\sim 1 \mathrm{nA} /$ strip for two SSDs and $\sim 3 \mathrm{nA} /$ strip for three SSDs. The two detectors with the lower leakage current were tested in CERN lead primary and fragmented beam in 2016 and the results were reported in Ref. [7]. In the 2018 beam test, the performance of one of the SSDs with $\sim 3 \mathrm{nA} /$ strip was characterized.

\section{2018 CERN Beam Test Configuration}


Figure 3: A schematic of the CERN test beam experimental setup used to determine the silicon detector charge resolution performance.

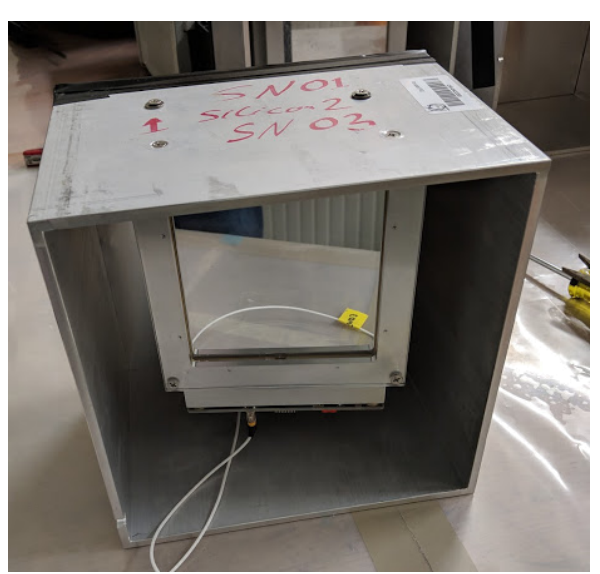

Figure 4: The mounting of two calibration silicon detectors in their lightbox.

The CERN beam test occurred November 21, 2018 to December 2, 2018 in the H8A beamline in the North Area of CERN. The primary beam consisted of ${ }^{208} \mathrm{~Pb}$ nuclei with $150 \mathrm{GeV} /$ nucleon kinetic energy. Fragmented beams with $A / Z=2.4,2.2, \& 2.0$ were used to determine the SSD response from helium $(Z=2)$ through lead $(Z=82)$. While several experimental configurations were employed during the CERN test beam run, we report on the results from the final configuration that achieved the best charge resolution performance of the silicon detectors, especially at the lowest atomic numbers $(Z \lesssim 10)$. The schematic of this experimental setup is shown in Figure 5. The HNX prototype silicon strip detector (pSSD) under test was mounted in a separate lightbox and placed behind four calibration silicon detectors (cSDs), with two upstream and two far downstream of the pSSD. This was followed an experiment that assessed the performance of silicon photomultiplier (SiPM) versus standard PMT readout of a Cherenkov detector with aerogel radiators followed by a Cherenkov detector with acrylic radiators. The Cherenkov lightboxes were followed by a lightbox with a plastic scintillators used in the beam trigger. Next a prototype Advanced Particle-astrophysics Telescope (APT) detector's response to heavy nuclei was characterized by researchers from Washington University in St. Louis. Next, the lightbox with two downstream cSDs was located immediately behind the WUSTL APT prototype 
detector in order to tag non-interacting nuclei in the beam for this experiment. Finally, a lightbox used for a SiPM beam radiation exposure ended the stack of instruments. The results of the SiPM Cherenkov and irradiation experiments are reported in a separate paper in this conference [8].

The cSDs were $\sim 10 \times 10 \mathrm{~cm}^{2}, 500 \mu \mathrm{m}$ thick pad detectors with the ohmic side of each detector read out separately. The 32 strips on the HNX pSSD were electrically connected together with the junction and ohmic sides read out individually. Figure 4 shows two cSDs mounted in their lightbox before the cover was attached, and the HNX pSSD was similarly mounted in its own lightbox. The cSDs and pSSDs were mounted at a $10^{\circ}$ angle with respect to the beam direction in order to minimize any crystal channeling effects. The cSDs and pSSD were AC-coupled, using a $0.1 \mu \mathrm{F}$ capacitor, to RL-724 (manufactured by Rel-Labs) charge preamplifiers $\left(\mathrm{C}_{\text {feedback }}=33 \mathrm{pF}\right.$ ) with each coupled to Canberra 2022 shaping amplifiers (using $1 \mu$ s shaping times) whose signals were then supplied to the inputs of a SuperTIGER FEE boards interfaced to the SuperTIGER data acquisition (STDAQ) system [6]. Each FEE channel of the STDAQ amplifies the input signal using separate lo-gain and hi-gain channels with a $\times 8$ gain difference. The output of each of the gain channels is digitized by 16-bit ADCs, yielding an effective ADC range of 19 bits for each FEE input. The polarity of the the shaping amplifier signal was adjusted to provide positive polarity signals, for both the ohmic and junction side readouts, into the SuperTIGER FEE input channels since the FEE requires a positive unipolar signal. The cSDs and pSSDs were biased at positive 60 volt depletion voltage on the ohmic sides during all tests. The guard rings and field plates of the pSSD were grounded for all tests.
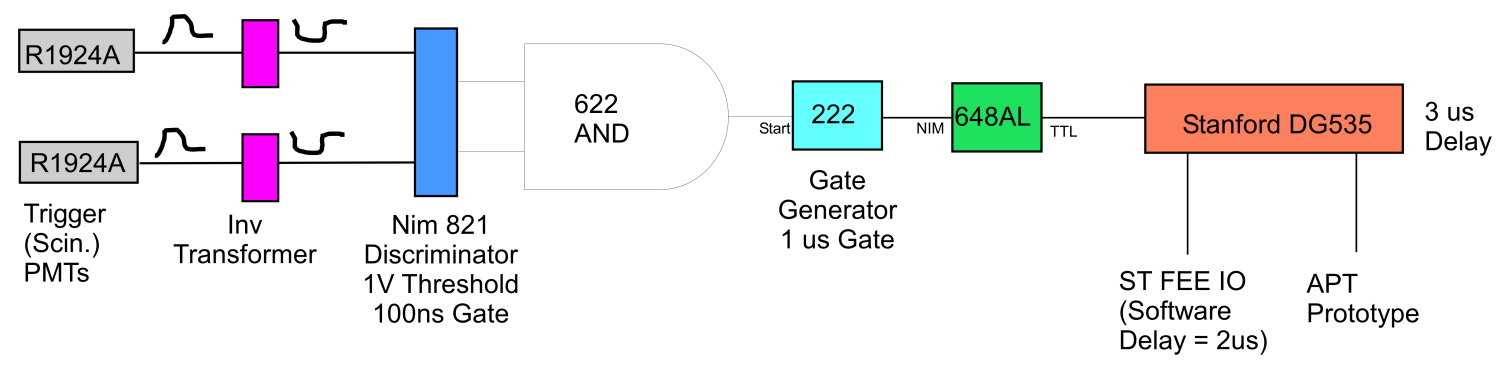

Figure 5: A schematic of the beam trigger used in the CERN test beam experiments.

The data acquisition system required a beam trigger that was delayed by $\sim 3 \mu$ s after a beam particle's passage through the stack of detectors. The trigger needed to be moderately efficient for charges $1 \leq Z \leq 82$, corresponding to the range of fragments available in the beam. The beam rate was adjusted to $\sim 1 \mathrm{kHz}$, in order that pileup was not an issue. In addition, fast timing was not a critical design parameter. The trigger used a coincidence between the Hamamatsu R1924 PMTs on the two integrating Cherenkov boxes (acrylic and aerogel) after the PMT signals were split between the acquisition system and the trigger. Inverting transformers converted the positive polarity PMT signals on the trigger path to negative polarity. The signals were then discriminated ( $\sim 1 \mathrm{~V}$ threshold on a LeCroy 821, $100 \mathrm{~ns}$ widths) and a coincidence generated by a LeCroy 622 logic module. The NIM-level signal started a gate generator set to a $1 \mu \mathrm{s}$ width that was converted by a LeCroy 688AL level converter to TTL. The TTL gate went to a Stanford DG535 gate generator set to generate the trigger TTL pulse after a $3 \mu$ s delay. Based on the complete range of charges seen by the silicon strip detectors, the trigger met the charge detection efficiency design goal. 


\begin{tabular}{|l|c|c|c|c|}
\hline & Pure Pb & $\mathrm{A} / \mathrm{Z}=2.4$ & $\mathrm{~A} / \mathrm{Z}=2.2$ & $\mathrm{~A} / \mathrm{Z}=2.0$ \\
\hline Total Beam Triggers & $1.2 \times 10^{5}$ & $1.6 \times 10^{5}$ & $5.0 \times 10^{5}$ & $3.1 \times 10^{5}$ \\
\hline Analyzed Events & $2.2 \times 10^{4}$ & $3.2 \times 10^{4}$ & $1.3 \times 10^{5}$ & $1.2 \times 10^{5}$ \\
\hline Fraction Analyzed & $17 \%$ & $20 \%$ & $25 \%$ & $39 \%$ \\
\hline
\end{tabular}

Table 1: Test beam event statistics for the runs taken on Sunday Dec. 2, 2018.

\subsection{Beam Test Results: Charge Resolution Analysis}

The set of primary and fragmented lead beam runs that were used in the analysis as well as the run statistics are summarized in Table 1. Pedestal runs occurred before and during the runs between beam spills and were subtracted to obtain the measurement of the ionization energy in each silicon detector. For the test beam runs used in this analysis, the width of the pedestals were $\sigma \approx 0.13$ MIP units for the cSDs and $\sigma \approx 0.1$ MIP units for both the ohmic and strip sides of the HNX pSSD. Here we present the details of the silicon detector charge resolution analysis.

Charge identification using the Calibration Silicon Detectors (cSDs) Using the information from the upstream and downstream cSDs, the particle charge is identified and non-interacting events are selected. Figure 6 is the charge distribution of two upstream layers of cSDs. The charge resolution of cSDs is determined to be $0.22 e$ with using the first two CSDs and 0.19 with all four layers. The charge consistency of all layers within the $\left|Z_{i}-Z\right|<1(i=1-4)$, where $i$ is the ID of cSDs, are required for the HNX pSSD analysis.

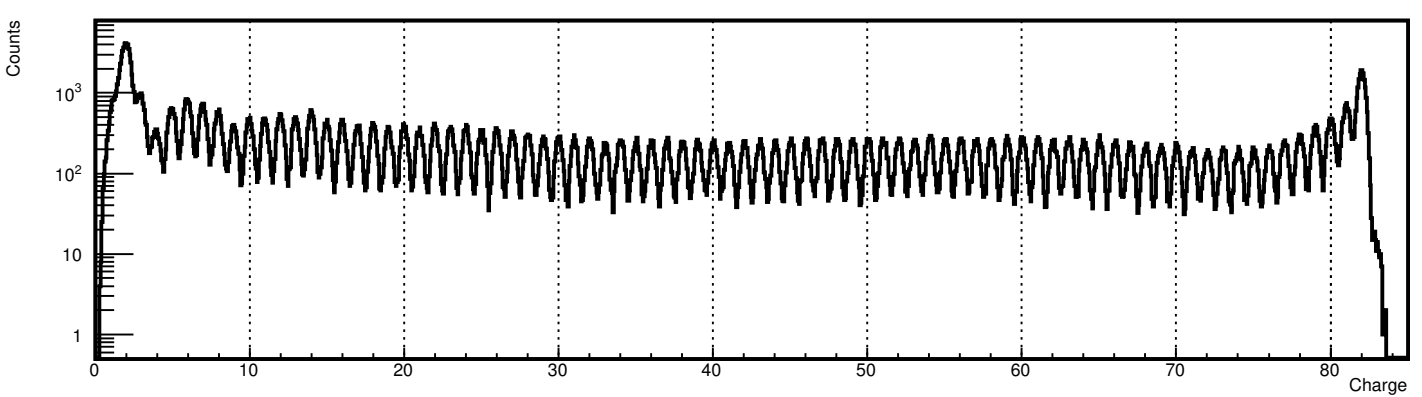

(a) $Z=2-82$



(b) $Z=2-16$



(c) $Z=70-82$

Figure 6: Charge distributions of first two upstream layers of calibration silicon detectors using the charge selection defined by the four cSDs. Each color histograms show the events identified by the 4 layers of cSDs. 
Calibration of the HNX prototype Silicon Strip Detector (pSSD) Using the events tagged the charge by 4 layers of cSDs, the detector response is calibrated. At first, the raw ADC signal is normalized at the gaussian peak of $\mathrm{Pb}$. Then, the all gaussian peaks are obtained in MIP units as shown in Fig. 7. As shown in Fig. 7, the linearity is remarkable throughout the range $2 \lesssim$ $Z \leq 82$ within $0.4 \%$ for both ohmic-side and strip-side for $Z \gtrsim 5$. After the correction of the linearity, the particle charge with HNX-Silicon detector is calculated as $Z=\sqrt{Q}$, where $Q$ is the MIP. Figure 8 and 9 show the charge distribution of HNX pSSD with ohmic side and strip side analyzed separately. The charge resolution of HNX pSSD shows in Fig. 10. The resolution is constant with $0.24 e$ in the range of $Z \gtrsim 5$ for both the ohmic and strip side results.

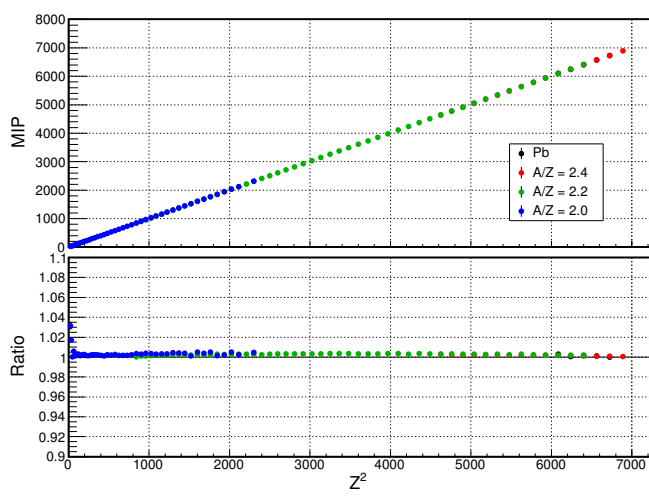

(a) Ohmic-side

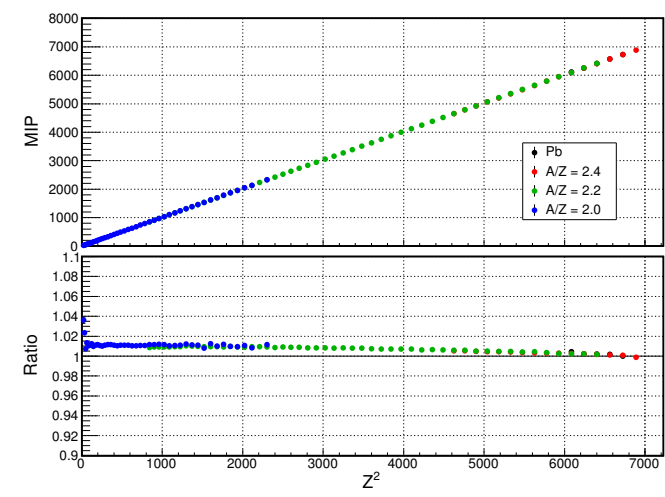

(b) Strip-side

Figure 7: The linearity of the HNX prototype Silicon Strip Detector as a function of $Z^{2}$ which is normalized by using $\mathrm{Pb}$ peak.

\section{Discussion}

The beam test results demonstrate that the HNX prototype silicon strip detectors can achieve 0.25 charge units of resolution for $5 \gtrsim Z \leq 82$ based on the 2018 CERN test beam results, assuming sufficient performance of the read out electronics. This is consistent with the results we obtained on different HNX pSSDs in the 2016 CERN test beam run [7], except we were able to reduce the effects of electronic noise in the 2018 run while running with higher beam rates, which allowed a better measurement of the charge resolution below $Z \gtrsim 10$. We note that individual elements from helium through lead are clearly resolved, with the modest reduction in the charge resolution for $Z \lesssim 5$ shown in Figure 10. The resolution degradation is consistent being due to the inherent noisy environment associated with being in an accelerator test beam in terms of the charge selection requirements used in the analysis. As shown in Figure 7, the uncorrected linearity of the HNX is remarkable for both the ohmic and strip side readout of the pSSD. Finally, we note that the ability to use the SuperTIGER FEE boards and DAQ system with the input signals defined by the output of shaping amplifiers aided in obtaining the superb charge resolution and is an innovative way to use this system. The FEE input channels each use shaping amplifiers with $1 \mu$ s time-to-peak which are then processed by peak-sensitive ADCs [6]. Normally, the output of a charge preamplier would provide the input signals to the FEE boards. 


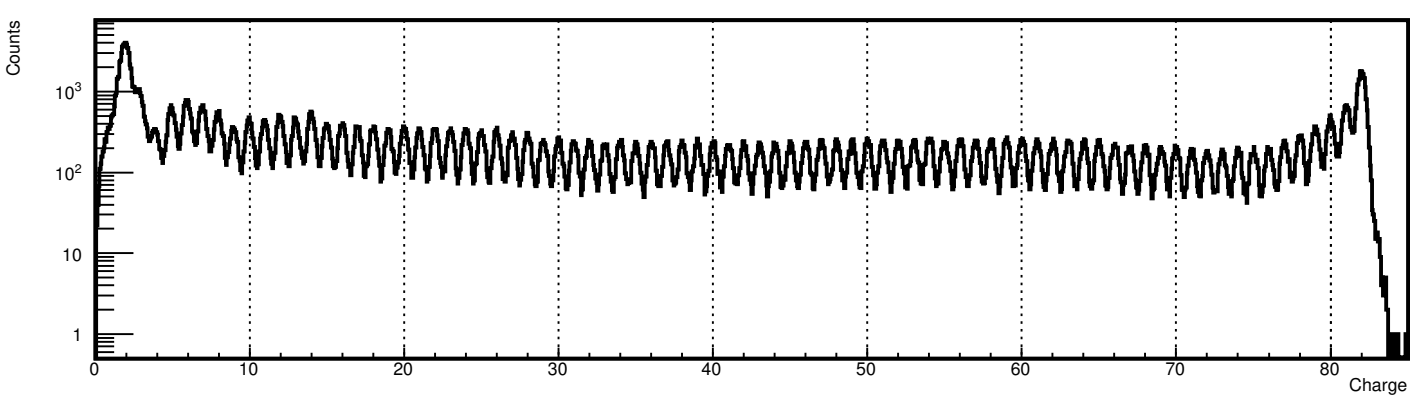

(a) $Z=2-82$

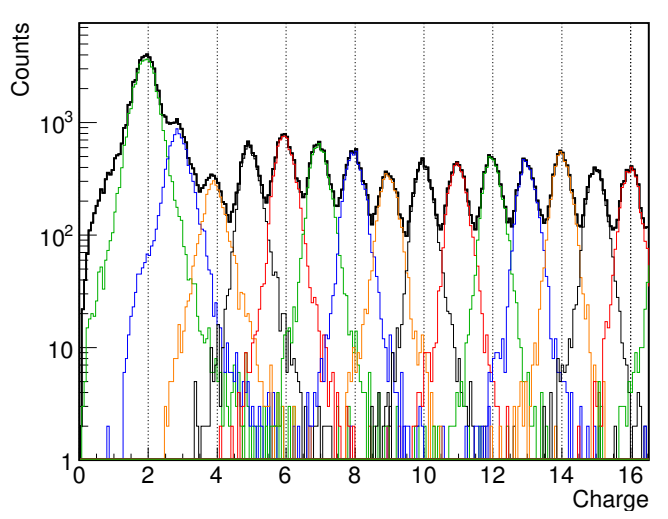

(b) $Z=2-16$

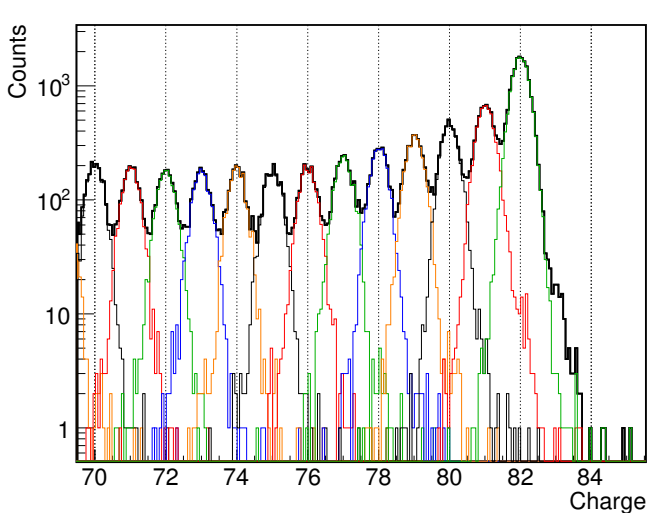

(c) $Z=70-82$

Figure 8: Charge distributions of ohmic side of a single HNX pSSD using the charge selection defined by the four cSDs. Each color histograms show the events identified by the 4 layers of cSDs.

\section{Acknowledgements}

We thank Mark Wiedenbeck (CalTech/SRL/JPL) for the use of the silicon calibration detectors, the Rel-Labs preamplifier electronics, and the Canberra shaping amplifiers used in this beam test. We also thank Marco Ricci (INFN/Frascati) for help in obtaining electronics and supplies from the CERN electronics pool. Finally, we thank CERN for the opportunity to perform this beam test and providing exceptional particle beams.

\section{References}

[1] J.W. Mitchell, Proc of the $36^{\text {th }}$ ICRC (Madison), PoS(ICRC2019)109 (2019)

[2] T. Hams, et al., Proc of the $34^{\text {th }}$ ICRC (The Hague), PoS(ICRC2015)038 (2015)

[3] W.R. Binns et al., Proc of the $31^{\text {st }}$ ICRC (Łódź), Paper 0441 (2009)

[4] B.A. Weaver et al., NIMB, Vol 145, 3, p. 409-428 (1998)

[5] R.A. Mewaldt et al., Space Sci. Rev., Vol 136, 1-4, p. 285-362 (2008)

[6] W.R. Binns et al., ApJ, Vol. 788, id. 18 (2014)

[7] J.F. Krizmanic et al., Proc of the $35^{\text {th }}$ ICRC (Buson), PoS(ICRC2017)242 (2017)

[8] J. Link et al., Proc of the $36^{\text {th }}$ ICRC (Madison), PoS(ICRC2019)096 (2019) 


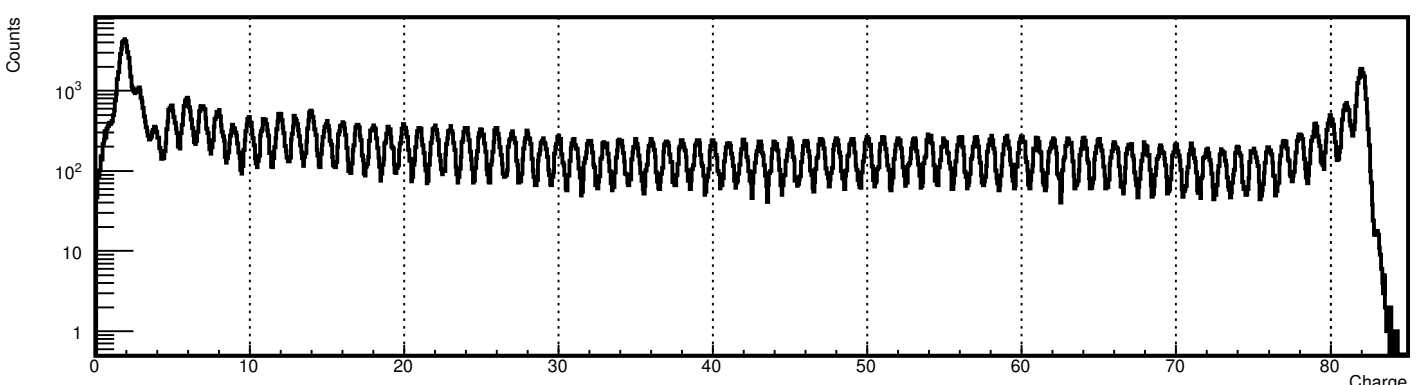

(a) $Z=2-82$

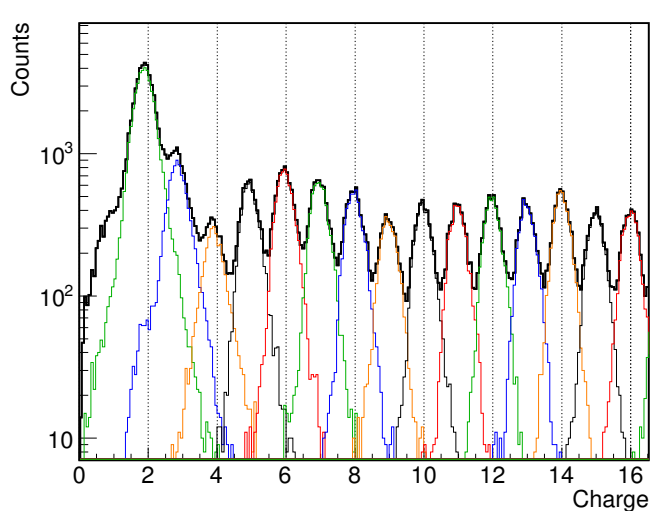

(b) $Z=2-16$

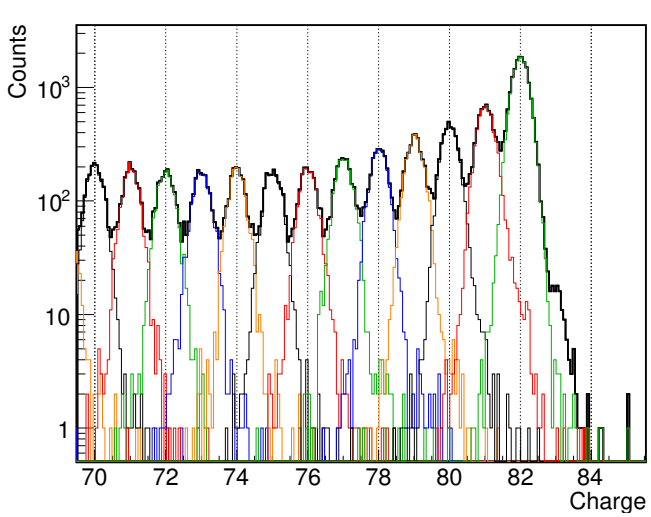

(c) $Z=70-82$

Figure 9: Charge distributions of strip side of of a single HNX pSSD using the charge selection defined by the four cSDs. Each color histograms show the events identified by the 4 layers of cSDs.

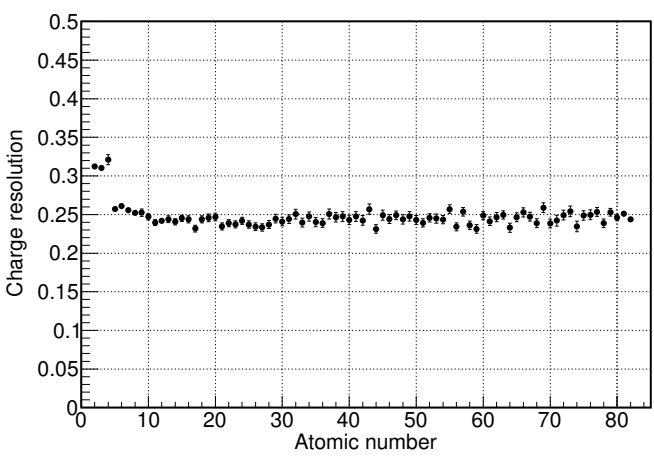

(a) Ohmic-side

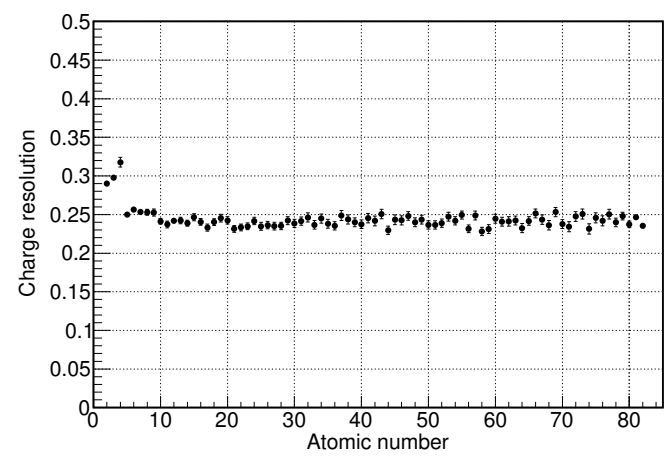

(b) Strip-side

Figure 10: The charge resolution of a single HNX pSSD. 